\title{
Development of a Scale to Screen Parents with Uncertainty Regarding Their Child with Acute Illness
}

\author{
Shingo Ueki', Kazuyo Komai², Kazutomo Ohashi ${ }^{3}$ \\ ${ }^{1}$ School of Nursing, Mukogawa Women's University, Hyogo, Japan \\ ${ }^{2}$ Nakano Children's Hospital, Osaka, Japan \\ ${ }^{3}$ Division of Health Science, Graduate School of Medicine, Osaka University, Osaka, Japan \\ Email: ueki@mukogawa-u.ac.jp
}

How to cite this paper: Ueki, S., Komai, K. and Ohashi, K. (2017) Development of a Scale to Screen Parents with Uncertainty Regarding Their Child with Acute Illness. Open Journal of Nursing, 7, 1246-1257. https://doi.org/10.4236/ojn.2017.711090

Received: October 27, 2017

Accepted: November 17, 2017

Published: November 20, 2017

Copyright $\odot 2017$ by authors and Scientific Research Publishing Inc. This work is licensed under the Creative Commons Attribution International License (CC BY 4.0).

http://creativecommons.org/licenses/by/4.0/

\begin{abstract}
Parents experience uncertainty when their children become sick. The study aimed to develop a Parents' Uncertainty regarding their Child with Acute Illness Scale (PUCAS) and to clarify differences in PUCAS scores between groups that were divided according to participants' demographic characteristics. PUCAS was developed based on interviews, literature review, and a pilot study. We obtained valid responses from 235 parents with children hospitalized due to an acute childhood illness. Exploratory factor analysis narrowed the number of items to 25 , divided into the following 5 subscales: unpredictability of the course of the illness, ambiguity about the severity of the illness, ambiguous appropriateness of management, discrepancy of judgement with health care professionals, and lack of information about the causes of the illness. There was satisfactory construct validity and criterion-related validity. Cronbach's alpha was 0.92 for the overall scale. Participants who used ambulances, those who were not given any reliable diagnosis for their children, and fathers scored significantly higher on the PUCAS. PUCAS has high validity and reliability in measuring uncertainty of parents who have children with acute childhood illness and could be a useful screening tool for parents with high uncertainty in a clinical setting.
\end{abstract}

\section{Keywords}

Acute Childhood Illness, Factor Analysis, Parent, Scale Development, Pediatric Nursing, Uncertainty

\section{Introduction}

Acute childhood illnesses (ACIs, e.g., respiratory infectious diseases and infectious 
gastroenteritis), defined as sudden onset and acute recovery from illness in childhood [1], are common among children up to two years old. Although mainly self-limiting, ACIs are a significant cause of concern and uncertainty regarding long-term damage or death for parents [2] [3] [4]. Parents often take their children to the doctor because of uncertainty about their child's illness rather than because they believe their child has a definite health problem [5]. Many parents, particularly those with less experience, lack confidence in differentiating serious and/or treatable infections from self-limiting ones [5]. When an ill child is hospitalized, parents struggle to cope with uncertainty [6]. Therefore, parents experience uncertainty from the onset until the child's hospitalization.

Mishel [7] conceptualized uncertainty theory, defining it as a cognitive situation in which a decision maker is unable to assign definite values to events about an illness or to accurately structure or classify the illness because of insufficient cues regarding the events. This is important when considering support for patients and their families [8]. Based on Mishel's theory, several scales were developed to measure the uncertainty of various groups such as adult patients [9], child patients [10], and parents with an ill child [7]. Of these scales, the Parents' Perception of Uncertainty Scale [7] (PPUS) to measure parents' uncertainty was translated into many languages and is used around the world. Thus, uncertainty has been measured under various conditions and standardized as an outcome measure for interventions [11]. However, the diseases for which these uncertainty scales are suitable are chronic illnesses, and a scale for uncertainty of parents with an acutely ill child has not been developed. Acute childhood illness, in contrast to chronic illness, shows significant changes in symptoms and recovery is expected. We clarified that parents who have a child with ACI felt uncertainty, which composed different from those with chronically ill children [12].

An important problem for current pediatric healthcare that has been reported is a lack of health information sought by uncertain parents. Most parents reported searching for health information on the internet but could find little helpful information [13]. Over half of the parents reported one or more unmet information needs for guidance or education from medical staff [14]. About 23\% of pediatricians do not ask open-ended questions to solicit parental concerns, but over half of parents or children cannot ask about their own concerns when their pediatricians do not ask the relevant questions [15]. This lack of information was reported as one of the component factors of parents' uncertainty [7]. The uncertainty of parents having children with ACI leads to negative effects such as rejection of treatment [16], worsening of the child's condition [17] [18] [19], and parents' depression [20]. Therefore, assessing which parents are uncertain is important for pediatric healthcare.

This study aimed to develop a Parents' Uncertainty regarding a Child with Acute Illness Scale (PUCAS) with sufficient validity and reliability using exploratory factor analysis. This scale would be useful as an assessment tool to screen 
for parents with high uncertainty. Such screening would help busy healthcare professionals provide additional explanations selectively and efficiently to uncertain parents in clinical settings. As the purpose of using this scale was screening, we needed to establish that parents in more uncertain situations had high uncertainty in clinical settings. Therefore, as a second aim, we intended to determine the differences in PUCAS scores between various subgroups divided according to the participants' demographic characteristics.

\section{Methods}

\subsection{Participants}

Parents whose child was hospitalized for an ACI at a children's hospital were invited to participate in the present study. This hospital specializes in pediatric internal medicine and is certified as a pediatric emergency unit of a secondary care facility in urban Japan. The definition of ACI was childhood illness with rapid onset, short course, and evident symptoms including upper respiratory tract infections, childhood infectious diseases, gastroenteritis, acute exacerbations for chronic conditions such as asthma, or other febrile illness [1].

\subsection{Ethical Considerations}

Prior to data collection, we obtained approval from the Research Ethics Committee at Osaka University (No. 15213) and the Institutional Review Board of the research hospital (No. 23). All participants were given a participant information sheet that included the purpose and method of the study, privacy protection, and a statement that participation was free with no penalty for non-participation. Parents who agreed to participate in this research were given an anonymous questionnaire and an institutional review board-approved book token with 500 yen (approximately US \$4.76) to encourage a higher response rate. We considered parents who responded to the questionnaire to be the study participants.

\subsection{Item Generation}

The development of PUCAS was conducted in the following phases.

\subsubsection{Phase 1: Interviews}

The first author conducted open-ended interviews with fifteen mothers of hospitalized children with ACI individually and clarified their uncertainty [12]. The codes in this interview data were replaced with questions.

\subsubsection{Phase 2: Literature Review}

We reviewed the literature to gather information about parents' psychological state when their child had an ACI. In this phase, we revised and added the question sentences from phase 1 and developed a first draft of PUCAS consisting of 37 items. Each item was rated on a five-point Likert style scale $(1=$ strongly disagree to $5=$ strongly agree). 


\subsubsection{Phase 3: A Pilot Study and Consultation}

Ten mothers whose child had had an ACI in the past were recruited for the pilot study. Mothers were asked to respond to a draft of PUCAS. We asked mothers for comments on the understandability and applicability of the questions, and revised some question sentences and omitted one inadequate item according to their comments. We also received feedback as we were supervised by an expert in adult patients' uncertainty in Japan, and revised the question sentences. The final draft of PUCAS consisted of 36 items.

\subsection{Data Collection}

We distributed an anonymous questionnaire and information sheet to parents within 24 hours after hospitalization. Participants deposited the completed questionnaire in a lockbox on the ward. To achieve adequate power for exploratory factor analysis, the sample size was estimated to require at least 200 valid responses [21]. We predicted approximately $70 \%$ would be valid responses, so we distributed 280 questionnaires to participants.

\subsection{Instruments}

The anonymous questionnaire included participants' demographic characteristics, the final draft of PUCAS, the State-Trait Anxiety Inventory (STAI), the Short Form of the Profile of Mood States (POMS), and open-ended questions. Demographic characteristics included the participant's age and sex, worker (yes or no), their child's age, the child's diagnosis, the estimated hospitalization period, use of ambulance when being transported to the hospital (yes or no), and experience of hospitalization in the past. We used the STAI and POMS to test for correlations with PUCAS to establish criterion-related validity. These scales have significant correlations with uncertainty scales of adult patients or parents [8] [22] [23]. We received copyright permission for use of the STAI and POMS questionnaires. Open-ended questions in the questionnaire asked about the contexts of uncertainty when parents recognized their child's symptoms, when parents took their child to hospital for the first consultation, and when their child was hospitalized. Data on time of visiting hospital (out of office hours or office hours) and referrals from other medical institutions were collected from the clinical records.

\subsubsection{State-Trait Anxiety Inventory}

The STAI [24] is a validated and widely used self-report measure of anxiety. The STAI consists of two subscales-the State Anxiety Form (SAF) and Trait Anxiety Form (TAF). The SAF measures an individual's state of anxiety at a particular moment, and the TAF measures an individual's personality trait of anxiety. Each form consists of 20 items and each item score ranges from 1 to 4 . The range of each form score is $20-80$, with a higher score indicating greater anxiety. The validity and reliability of the Japanese version of the STAI has also been confirmed [25]. 


\subsubsection{Profile of Mood States}

The Short Form of the Profile of Mood States [26] is a self-administered test that identifies and assesses transient and fluctuating affective states in individuals. The test assesses the following six components of mood: tension-anxiety (T-A), depression (D), anger-hostility (A-H), fatigue (F), confusion (C), and vigor (V) subscales. Each subscale includes five items. Answers ranges from 0 (not at all) to 4 (extremely) on a five-point scale. In this study, the T-A, D, and C subscales were used to evaluate correlations with the PUCAS. The validity and reliability of these POMS subscales was confirmed using a Japanese sample [27].

\subsection{Data Analysis}

Data analysis was conducted using SPSS version 21.0 (IBM, Tokyo, Japan). Exploratory factor analysis was performed to examine the construct validity of the PUCAS. The number of factors was determined based on the eigenvalue greater than 1.0 rule, examination of a scree plot, and/or their conceptual meanings [28]. We assessed the conceptual meanings based on the results of content analysis in phase 1 of the study. Item analysis was conducted for inter-item correlations and item-total correlation by using Pearson's correlation coefficients. Inter-item correlations of $0.30-0.80$ and item-total correlations of $0.20-0.80$ were considered satisfactory [29]. The criteria for omitting items were as follows: presence of a ceiling effect or floor effect; structure coefficients, i.e., correlations of each item with the total score of the factor less than 0.40; factor loadings less than 0.40; and inter-item correlations and item-total correlations out-of-range as described above. We conducted factor analysis repeatedly while one of each item excluded by these criteria was omitted because eigenvalues and factor loadings would change if any one item was omitted. We completed the analysis when a solution was attained in which all the items included in the analysis met all criteria. For descriptive analysis, inter-correlations among the factors and correlation between each factor and full scale score were also calculated. Criterion-related validity was examined by calculating Pearson's correlations between the PUCAS total and factor scores and the SAF, TAF, T-A, D, and C. The internal consistency and reliability of the PUCAS was assessed using Cronbach's alpha (alpha); a value greater than 0.7 was taken as satisfactory. As the second aim of the present study, we conducted unpaired t-tests to clarify the differences in PUCAS scores between groups divided by the following five kinds of participant demographic characteristics: sex of participants (mother or father), visiting time period (out of office hours or office hours), referral from other medical institutions (yes or no), use of ambulance (yes or no), experience of hospitalization in the past (yes or no), and diagnosis given for their children (yes or no). A p value of $<$ 0.05 indicated statistical significance.

\section{Results}

We distributed 280 questionnaires to participants from November 2015 to 
February 2016, of whom 24 did not respond and 21 did not respond completely. A total of 235 (83.9\%) provided valid responses. The mean age of the participants was 34.5 years (standard deviation $[\mathrm{SD}]=5.7$ ). The major participant characteristics were mother $(220 ; 93.6 \%)$, first time of hospitalization (147; 62.6\%), not using an ambulance $(205 ; 87.2 \%)$, and workers $(122 ; 51.9 \%)$. Mean age of the child was 37.7 months $(\mathrm{SD}=38.3)$. The most common children's illness was respiratory infection $(109 ; 46.4 \%)$. Twenty-nine children did not receive any diagnosis. One-hundred-eighty-six parents were informed by the doctor of the expected hospitalization period, which on average was 7.0 days. Responses to the open-ended questions inquiring as to the contexts of parents' uncertainty were similar to the context of items of the PUCAS and did not include any new uncertainties.

\subsection{Construct Validity}

Before analysis, we omitted one item because of a floor effect. After that, exploratory factor analysis was conducted for verification of construct validity on the PUCAS while implementing the major factor method and promax rotation. According to the results of the factor analysis, less than eight factors had an eigenvalue of more than 1.0 and six factors were suitable based on results of the scree plot. Of the 35 items, three items that showed inter-item correlations of more than 0.8 and two items that showed inter-item correlations of less than 0.3 were omitted. After these items were omitted, the eigenvalues and scree plot changed and five factors were suitable. We repeatedly analyzed while omitting one of each item if the factor loading of the item was less than 0.4. Finally, the factor loading of each of the final 25 items was shown to be more than 0.4 , the structure coefficients were more than 0.54 , inter-item correlations ranged from 0.32 $0.71(\mathrm{p}<0.01)$, and item-total correlations ranged from $0.37-0.73(\mathrm{p}<0.01)$, satisfying the criteria. Total variance explained before rotation was $63.3 \%$. The results of factor loadings of each item and names of each factor are shown in Table 1. Item analysis is shown in Table 2. Inter-correlations were all significant and each factor significantly correlated with the total PUCAS $(\mathrm{p}<0.01)$ (Table 2).

\subsection{Criterion-Related Validity}

To determine the criterion-related validity of the PUCAS, we analyzed the correlations of the PUCAS total and factors scores with the SAF, TAF, T-A, D, and C (Table 3). The PUCAS total and factor scores showed positive correlations with all scales. However, correlations of the PUCAS (total and factors scores) with the TAF were small or not significant.

\subsection{Internal Consistency Reliability}

The internal consistency of the overall scale was high (alpha $=0.92$ ), with good subscale reliability (alpha $=0.79-0.87)$ as shown in Table 1 . 
Table 1. Exploratory factor analysis results $(\mathrm{n}=235)$.

\begin{tabular}{|c|c|c|c|c|c|}
\hline Factors & \multicolumn{5}{|c|}{ Factor loading } \\
\hline I cannot imagine what life after recuperation will be like & 0.95 & 0.01 & -0.13 & -0.04 & -0.08 \\
\hline I cannot predict the course of my child's illness & 0.68 & 0.13 & -0.07 & 0.01 & 0.05 \\
\hline I cannot predict how long the state of my child's illness will last & 0.63 & 0.04 & -0.03 & -0.01 & 0.07 \\
\hline I cannot imagine how I will manage the care of my child. & 0.62 & -0.14 & 0.18 & 0.08 & 0.06 \\
\hline I am not sure how I can make my child comfortable & 0.59 & -0.02 & 0.26 & 0.01 & 0.03 \\
\hline \multicolumn{6}{|l|}{ Factor 2: Ambiguity about the severity of the illness } \\
\hline I cannot determine the seriousness of my child's illness & -0.11 & 0.86 & -0.07 & -0.01 & 0.08 \\
\hline I am unsure what is normal for my child. & -0.02 & 0.81 & -0.05 & -0.17 & 0.00 \\
\hline I am unsure if my child's illness is getting better or worse. & 0.20 & 0.55 & -0.13 & 0.13 & -0.02 \\
\hline It is unclear if my child's behavior is because of some symptoms & -0.06 & 0.54 & 0.12 & 0.12 & 0.08 \\
\hline It is unclear what is happening to my child & 0.22 & 0.52 & 0.02 & -0.01 & 0.08 \\
\hline It is difficult to understand what my child wants & 0.08 & 0.46 & 0.12 & 0.04 & -0.21 \\
\hline I don't know where to look to determine the state of my child's illness & 0.08 & 0.46 & 0.21 & 0.10 & -0.05 \\
\hline \multicolumn{6}{|l|}{ Factor 3: Ambiguous appropriateness of management } \\
\hline I am not sure how much attention I should pay to my child & -0.03 & 0.06 & 0.80 & -0.13 & 0.08 \\
\hline I don't know how much I should restrict my child's behavior & -0.06 & -0.11 & 0.77 & 0.02 & 0.11 \\
\hline I don't know which foods and drinks I should give to my child & -0.15 & 0.01 & 0.74 & 0.06 & -0.03 \\
\hline It is not clear when I should call for help & 0.23 & 0.02 & 0.55 & 0.03 & -0.18 \\
\hline I am not sure whether my care for my child is correct & 0.32 & 0.01 & 0.53 & -0.10 & 0.01 \\
\hline \multicolumn{6}{|l|}{ Factor 4: Discrepancy of judgement with health care professionals } \\
\hline It is difficult to know if the treatments my child is getting are helping & 0.02 & -0.01 & -0.04 & 0.90 & -0.04 \\
\hline I do not understand the necessity of continuing this treatment for my child & 0.09 & -0.07 & -0.17 & 0.85 & -0.03 \\
\hline I doubt the diagnosis my child received is true & -0.13 & 0.07 & 0.04 & 0.78 & 0.10 \\
\hline I doubt that this treatment is really suitable for my child's symptoms & -0.01 & -0.03 & 0.20 & 0.68 & 0.01 \\
\hline \multicolumn{6}{|l|}{ Factor 5: Lack of information about the cause of the illness } \\
\hline The places my child caught the illness seem hazy to me & 0.09 & -0.10 & -0.08 & -0.04 & 0.88 \\
\hline I don't know why my child became ill. & 0.14 & -0.03 & -0.05 & 0.07 & 0.73 \\
\hline I don't know from when my child became ill & -0.06 & 0.20 & 0.02 & -0.02 & 0.59 \\
\hline I think of different reasons why my child became ill & -0.14 & -0.01 & 0.20 & 0.02 & 0.56 \\
\hline Cronbach's alpha & 0.85 & 0.85 & 0.83 & 0.87 & 0.79 \\
\hline
\end{tabular}

\subsection{Comparison between Groups}

We calculated t-tests between groups divided by five participant demographic characteristics. Results are shown in Table 4. Of these five characteristics, participants who used ambulances, those who were not given any reliable diagnosis for their children, and fathers scored significantly higher on the PUCAS. 
Table 2. Correlations between items and groups of items.

\begin{tabular}{ccccccccc}
\hline & $\begin{array}{c}\text { Structure } \\
\text { coefficients } \\
\text { (range) }\end{array}$ & $\begin{array}{c}\text { Inter-item } \\
\text { correlation } \\
\text { (range) }\end{array}$ & $\begin{array}{c}\text { Item-total } \\
\text { correlation } \\
\text { (range) }\end{array}$ & F.1 & F.2 & F.3 & F.4 & $\begin{array}{c}\text { Inter-correlation } \\
\text { full scale }\end{array}$ \\
\hline F.1 & $0.66-0.83$ & $0.40-0.69$ & $0.62-0.73$ & - & & & & 0.84 \\
F.2 & $0.54-0.77$ & $0.32-0.60$ & $0.50-0.68$ & 0.65 & - & & & 0.84 \\
F.3 & $0.67-0.78$ & $0.41-0.58$ & $0.48-0.62$ & 0.62 & 0.55 & - & & 0.74 \\
F.4 & $0.76-0.87$ & $0.53-0.71$ & $0.50-0.62$ & 0.50 & 0.55 & 0.45 & - & 0.68 \\
F.5 & $0.56-0.86$ & $0.38-0.69$ & $0.37-0.50$ & 0.36 & 0.27 & 0.21 & 0.35 & 0.57 \\
\hline
\end{tabular}

F: Factor.

Table 3. Correlations of the total and factor score of the PUCAS with the subscales of the POMS and STAI.

\begin{tabular}{cccccc}
\hline & $\mathrm{D}$ & $\mathrm{T}-\mathrm{A}$ & $\mathrm{C}$ & $\mathrm{SAF}$ & $\mathrm{TAF}$ \\
\hline Factor 1 & $0.42^{*}$ & $0.36^{*}$ & $0.35^{*}$ & $0.36^{*}$ & $0.18^{*}$ \\
Factor 2 & $0.39^{*}$ & $0.34^{*}$ & $0.30^{*}$ & $0.26^{*}$ & $0.19^{*}$ \\
Factor 3 & $0.40^{*}$ & $0.40^{*}$ & $0.35^{*}$ & $0.29^{*}$ & 0.12 \\
Factor 4 & $0.30^{*}$ & $0.32^{*}$ & $0.30^{*}$ & $0.27^{*}$ & 0.08 \\
Factor 5 & $0.23^{*}$ & $0.22^{*}$ & $0.22^{*}$ & $0.21^{*}$ & $0.19^{*}$ \\
Total score & $0.48^{*}$ & $0.44^{*}$ & $0.44^{*}$ & $0.38^{*}$ & $0.22^{*}$ \\
\hline
\end{tabular}

$*$ : $<0.05$.

Table 4. Comparison of PUCAS between groups.

\begin{tabular}{|c|c|c|c|c|c|}
\hline & \multirow{2}{*}{$\mathrm{N}$} & \multicolumn{2}{|c|}{ PUCAS } & \multirow{2}{*}{$t$} & \multirow{2}{*}{$p$} \\
\hline & & Mean & $(\mathrm{SD})$ & & \\
\hline \multicolumn{6}{|l|}{ Sex of participants } \\
\hline Mother & 220 & 68.07 & $(18.16)$ & -2.33 & 0.02 \\
\hline Father & 14 & 79.71 & $(17.96)$ & & \\
\hline \multicolumn{6}{|l|}{ Time of visiting hospital } \\
\hline Office hours & 144 & 68.83 & $(19.78)$ & 0.07 & 0.94 \\
\hline Out of office hours & 91 & 68.66 & $(17.34)$ & & \\
\hline \multicolumn{6}{|l|}{ Referred from another hospital } \\
\hline Yes & 100 & 69.11 & $(17.88)$ & 0.19 & 0.85 \\
\hline No & 133 & 68.66 & $(18.70)$ & & \\
\hline \multicolumn{6}{|l|}{ Use of ambulance } \\
\hline Yes & 27 & 77.19 & $(17.11)$ & 2.53 & 0.01 \\
\hline No & 205 & 67.80 & $(18.27)$ & & \\
\hline \multicolumn{6}{|c|}{ Experience of hospitalization in the past } \\
\hline Yes & 86 & 69.23 & $(17.84)$ & 0.24 & 0.81 \\
\hline No & 147 & 68.63 & $(18.65)$ & & \\
\hline \multicolumn{6}{|c|}{ Diagnosis provided for their children } \\
\hline Yes & 203 & 67.83 & $(17.70)$ & -2.16 & 0.03 \\
\hline No & 29 & 75.62 & $(21.47)$ & & \\
\hline
\end{tabular}




\section{Discussion}

We attempted to develop a scale to measure parents' uncertainty when their children are hospitalized for ACIs. As a result, we produced the PUCAS consisting of 25 items in 5 subscales with sufficient validity and reliability. Higher scores on the PUCAS indicate greater parents' uncertainty.

We considered that the revealed factors had not lost any of the conceptual meanings based on the results of content analysis in phase 1 of the study. Especially, the PUCAS subscales "ambiguous appropriateness of management" and "lack of information about the cause of the illness" were particularly important in assessing the construct validity of the PUCAS. According to our qualitative study (Phase 1), we mentioned especially that these two categories were characteristic of perceptions among mothers with children who had ACIs. These components of uncertainties are important concern for parents. The criteria based on the factor analysis were all satisfactory, as shown in Table 1. The correlations between each item, factor, and the total score shown in Table 2 were all significant. Therefore, we believed that the PUCAS was adequately constructed. Regarding criterion-related validity, the correlations of the total and factor scores of the PUCAS with the STAI TAF were smaller than the other scales or not significant. The Universal Uncertainty in Illness Scale [8] (UUIS) which was used to measure adult patients' uncertainty correlated strongly with TAF, which differs from our result. Nogawa [8] reported that most participants measured by UUIS had chronic illness, and those with acute illnesses represented less than 3\% of the sample. In cases of chronic illness, patients need to live with the illness. In contrast, ACIs show rapid changes of symptoms and most symptoms recover eventually. Therefore, it is understandable that the PUCAS was affected more by the psychological state of anxiety at a particular moment than by trait anxiety, which is a feature of an individual's personality. The POMS subscales of T-A, D, and $\mathrm{C}$ showed moderate correlations $(\mathrm{r}>0.4 ; \mathrm{p}<0.05)$, indicating that the PUCAS had sufficient criterion-related validity. Regarding the reliability of the PUCAS, we decided that test-retest was inappropriate in this study because the child would recover by the time the retest was conducted. However, the Cronbach's alpha for internal consistency was greater than 0.7 in the overall scale and all subscales, indicating that the reliability was satisfactory.

The statistical tests of differences between groups revealed that parents who used ambulances, those who were not given any reliable diagnosis for their child, and fathers scored significantly higher on the PUCAS. Parents who used ambulances to transport their child to the hospital might have done so because they became upset when they saw their child suddenly worsen. The parents might not understand the information provided by medical staff just after hospitalization due to being in a panic, even if the staff explained carefully. In some cases, the children were not given any diagnosis because the children's symptoms had still not fulfilled certain diagnostic criteria or it had taken a long time to get test results. It is understandable that the parents experienced high uncertainty in predicting their children's illness course or treatment strategy in these cases. All 
fathers who participated in this study were workers. Therefore, the fathers might have accompanied their child temporarily when they were asked to participate by the researcher, and they might not have received explanations directly from medical staff. It was possible that the fathers did not sufficiently understand the state of their child's illness or treatment strategy although they might have been informed by the mothers. Thus, we can explain the reason for high uncertainty in the three cases described above. The PUCAS could measure the parents' uncertainty adequately in clinical setting.

Regarding study limitations, we recruited only Japanese parents as participants. It will be necessary to translate the PUCAS from Japanese into other languages and adapt it to country-specific health medical systems or cultural backgrounds that differ from Japan. Additionally, we could not examine the effects of parents' uncertainties on the parents themselves or parents' surroundings including their ill child. Parents of a child with a severe illness are likely to complain of post-traumatic stress syndrome [30]. The negative psychological stress including parents' anxiety or uncertainty could transfer to the ill child and potentially hinder the child's recovery [31] [32]. Reducing parents' uncertainty may lead to improvements in the child's health. A prospective study to disclose the effects of high parents' uncertainty will also be needed.

\section{Conclusion}

From our research regarding parents of a child hospitalized with an ACI, we achieved our goal of developing a scale named PUCAS that has sufficient validity and reliability to measure parents' uncertainty regarding their child's acute illness. The total PUCAS score was significantly higher in cases of participants who used an ambulance, those who were not given any reliable diagnosis for their children, and fathers. Therefore, the clinical validity of the PUCAS was also demonstrated. The PUCAS can be useful as an assessment tool to screen for parents with high uncertainty. We need to conduct further studies to examine the characteristics of parents' uncertainty in samples other than Japanese, to take into account country-specific health systems or cultural backgrounds. Understanding the effects of parents' uncertainty on the parents themselves or their surroundings including their ill child is also important.

\section{Acknowledgements}

We are particularly thankful to Dr. Minoru Kino for providing the investigation site and Prof. Michiko Nogawa for supervising the development of PUCAS. We also thank all the study participants.

\section{References}

[1] Neill, S.J. (2000) Acute Childhood Illness at Home: The Parents' Perspective. Journal of Advanced Nursing, 31, 821-832.

https://doi.org/10.1046/j.1365-2648.2000.01340.x 
[2] Garbutt, J.M., Leege, E., Sterkel, R., Gentry, S., Wallendorf, M. and Strunk, R.C. (2012) What Are Parents Worried about? Health Problems and Health Concerns for Children. Clinical Pediatrics, 51, 840-847. https://doi.org/10.1177/0009922812455093

[3] Cornford, C.S., Morgan, M. and Ridsdale, L. (1993) Why Do Mothers Consult When Their Children Cough? Family Practice, 10, 193-196. https://doi.org/10.1093/fampra/10.2.193

[4] Kai, J. (1996) Parent's Difficulties and Information Needs in Coping with Acute Illness in Preschool Children: A Qualitative Study. British Medical Journal, 313, 987-900. https://doi.org/10.1136/bmj.313.7063.987

[5] Cabral, C., Lucas, P.J., Ingram, J., Hay, A.D. and Horwood, J. (2015) "It's Safer to ...” Parent Consulting and Clinician Antibiotic Prescribing Decisions for Children with Respiratory Tract Infections: An Analysis across Four Qualitative Studies. Social Science \& Medicine, 136-137, 156-164. https://doi.org/10.1016/j.socscimed.2015.05.027

[6] Stratton, K.M. (2004) Parents Experiences of Their Child's Care during Hospitalization. Journal of Cultural Diversity, 11, 4-11.

[7] Mishel, M.H. (1983) Parents' Perception of Uncertainty Concerning Their Hospitalized Child. Nursing Research, 32, 324-330. https://doi.org/10.1097/00006199-198311000-00002

[8] Nogawa, M. (2012) Development of a Universal Uncertainty in Illness Scale to Be Used for Inpatients and Outpatients. Journal of Japan Academy Nursing Science, 32, 3-11. https://doi.org/10.5630/jans.32.1_3

[9] Mishel, M.H. (1981) The Measurement of Uncertainty in Illness. Nursing Research, 30, 258-263. https://doi.org/10.1097/00006199-198109000-00002

[10] Pai, A.L.H., Mullins, L.L., Drotar, D., Burant, C., Wagner, J. and Chaney, J.M. (2007) Exploratory and Confirmatory Factor Analysis of the Child Uncertainty in Illness Scale among Children with Chronic Illness. Journal of Pediatric Psychology, 32, 288-296. https://doi.org/10.1093/jpepsy/js1021

[11] Mullins, L.L., Fedele, D.A., Chaffin, M., Hullmann, S.E., Kenner, C., Eddington, A.R., et al. (2012) A Clinic-Based Interdisciplinary Intervention for Mothers of Children Newly Diagnosed With Cancer: A Pilot Study. Journal of Pediatric Psychology, 37, 1104-1115. https://doi.org/10.1093/jpepsy/jss093

[12] Ueki, S., Takao, K., Komai, K., Fujiwara, C. and Ohashi, K. (2017) Maternal Uncertainty for Acute Childhood Illness: A Qualitative Study. Open Journal of Nursing, 7, 645-656. https://doi.org/10.4236/ojn.2017.76048

[13] Moseley, K.L., Freed, G.L. and Goold, S.D. (2011) Which Sources of Child Health Advice Do Parents Follow? Clinical Pediatrics, 50, 50-56. https://doi.org/10.1177/0009922810379905

[14] Bethell, C., Reuland, C.H.P., Halfon, N. and Schor, E.L. (2004) Measuring the Quality of Preventive and Developmental Services for Young Children: National Estimates and Patterns of Clinicians' Performance. Pediatrics, 113, 1973-1983.

[15] Norlin, C., Crawford, M.A., Bell, C.T., Sheng, X. and Stein, M.T. (2011) Delivery of Well-Child Care: A Look Inside the Door. Academic Pediatrics, 11, 18-26. https://doi.org/10.1016/j.acap.2010.12.008

[16] Linnard-Palmer, L. and Kools, S. (2005) Parents' Refusal of Medical Treatment for Cultural or Religious Beliefs: An Ethnographic Study of Health Care Professionals' Experiences. Journal of Pediatric Oncology Nursing, 22, 48-57. https://doi.org/10.1177/1043454204270263 
[17] Francis, N., Wood, F., Simpson, S., Hood, K. and Butler, C.C. (2008) Developing an 'Interactive' Booklet on Respiratory Tract Infections in Children for Use in Primary Care Consultations. Patient Education and Counseling, 73, 286-293. https://doi.org/10.1016/j.pec.2008.07.020

[18] Neill, S.J., Cowley, S. and Williams, C. (2013) The Role of Felt or Enacted Criticism in Understanding Parent's Help Seeking in Acute Childhood Illness at Home: A Grounded Theory Study. International Journal of Nursing Studies, 50, 757-767. https://doi.org/10.1016/j.ijnurstu.2011.11.007

[19] Geldsetzer, P., Williams, T.C., Kirolos, A., Mitchell, S., Ratcliffe, L.A., Kohli-Lynch, M.K., et al. (2014) The Recognition of and Care Seeking Behaviour for Childhood Illness in Developing Countries: A Systematic Review. PLOS ONE, 9, e93427. https://doi.org/10.1371/journal.pone.0093427

[20] Franck, L.S., Wray, J., Gay, C., Dearmun, A.K., Lee, K. and Cooper, B.A. (2015) Predictors of Parent Post-Traumatic Stress Symptoms After Child Hospitalization on General Pediatric Wards: A Prospective Cohort Study. International Journal of Nursing Studies, 52, 10-21. https://doi.org/10.1016/j.ijnurstu.2014.06.011

[21] Comrey, A.L. and Lee, H.B. (2013) A First Course in Factor Analysis, 2nd Edition, Psychology Press, Hove.

[22] Taylor-Piliae, R.E. and Molassiotis, A. (2001) An Exploration of the Relationships Between Uncertainty, Psychological Distress and Type of Coping Strategy among Chinese Men after Cardiac Catheterization. Journal of Advanced Nursing, 33, 79-88. https://doi.org/10.1046/j.1365-2648.2001.01640.x

[23] Mu, P.F., Ma, F.C., Hwang, B. and Chao, Y.M. (2002) Families of Children with Cancer: The Impact on Anxiety Experienced by Fathers. Cancer Nursing, 25, 66-73. https://doi.org/10.1097/00002820-200202000-00012

[24] Spielberger, C.D. (1966) Anxiety and Behavior, Academic Press, New York.

[25] Curran, S.L., Andrykowski, M.A. and Studts, J.L. (1995) Short Form of the Profile of Mood States (POMS-SF): Psychometric Information. Psychological Assessment, 7, 80-83. https://doi.org/10.1037/1040-3590.7.1.80

[26] Hidano, T., Fukuhara, M., Iwawaki, S., Soga, S. and Spielberger, C.D. (2000) STAI Manual. Zitsumukyoiku-syuppan, Tokyo.

[27] Yokoyama, K. (2005) Japanese Version of POMS-Brief Form. Kaneko-shobo, Tokyo.

[28] Thompson, B. (2004) Exploratory and Confirmatory Factor Analysis: Understanding Concepts and Applications. American Psychological Association, Washington DC. https://doi.org/10.1037/10694-000

[29] Streiner, D.L. and Norman, G.R. (2003) Health Measurement Scales: A Practical Guide to Their Development and Use. Oxford University Press, New York.

[30] Santacroce, S.J. (2003) Parental Uncertainty and Posttraumatic Stress in Serious Childhood Illness. Journal of Nursing Scholarship, 35, 45-51. https://doi.org/10.1111/j.1547-5069.2003.00045.x

[31] Blake, J. (2006) Supporting Anxious Parents in Search of Internet Information. Nursing Times, 102, 24-26.

[32] Lester, K.J., Seal, K., Nightingale, Z.C. and Field, A.P. (2010) Are Children's Own Interpretations of Ambiguous Situations Based on How They Perceive Their Mothers Have Interpreted Ambiguous Situations for Them in the Past? Journal of Anxiety Disorders, 24, 102-108. https://doi.org/10.1016/j.janxdis.2009.09.004 